\title{
Stability Precision Error Correction of Photoelectric Detection by Unmanned Aerial Vehicle
}

\author{
Huajie Hong $\mathbb{D}^{\mathbb{B}}$, Keyan He $(\mathbb{D}$, Zihao Gan $(\mathbb{D}$, and Guilin Jiang $(\mathbb{D}$ \\ Unmanned System Institution, School of Intelligence and Technology, National University of Defense Technology, \\ Changsha 410073, China \\ Correspondence should be addressed to Keyan He; hekeyan@zju.edu.cn
}

Received 27 January 2021; Revised 7 March 2021; Accepted 13 March 2021; Published 5 April 2021

Academic Editor: Eduard Llobet

Copyright (c) 2021 Huajie Hong et al. This is an open access article distributed under the Creative Commons Attribution License, which permits unrestricted use, distribution, and reproduction in any medium, provided the original work is properly cited.

\begin{abstract}
For getting clear images and overcoming shaking caused by various disturbances, real-time compensation of pointing errors will improve the overall stability performance of photoelectric detection by unmanned aerial vehicle. However, the compensation will be greatly deteriorated by error-causing sources, and the error correction process is of great importance. In this research, the problem of stability precision error correction is comprehensively studied. First, by modeling overall kinematics, errorcausing sources, and error compensation, the error correction process is mathematically modeled and simulated. Then, by using simulation data regression, error correction models including the global function model and parametric model are established. The models are validated by carrying out both simulations and validation experiments. At last, the performances of the error correction models are compared and analyzed, which concerns the factors of parameter identification, model simplicity, and final improvement effect. Results show that the final stability precision can be greatly improved over $20 \%$, and the parametric model outperforms the global function model comprehensively. It can be concluded that, either in simulation environment or real application scenarios, the obtained models and related analysis results are effective in improving the system stability performance.
\end{abstract}

\section{Introduction}

To obtain clear images and overcome disturbances, stability precision (hereinafter, the precision) is a key index for evaluating performances of photoelectric detection devices, especially for lightweight and small-sized carriers like unmanned aerial vehicle (UAV), for those are easily affected by various disturbances and errors $[1,2]$. The typical application scenarios of this kind of device are shown in Figure 1.

Researchers have been always focusing on the stability precision control of photoelectric devices. The application scenarios include carriers like unmanned vehicles, aerial carriers, and naval vessels. For instance, Zhuchong et al. [3] developed an inertial-stabilized platform for airborne remote sensing using magnetic bearings. Zhou et al. [4] presented the design of a mechatronic system for a two-axis inertial-stabilized platform in an unmanned helicopter- (UH-) based airborne power line inspection system. Dong et al. [5] proposed a dynamic model and control method for a two-axis initially stabilized platform, and there are plenty of works in other fields concerning the problem of stability precession improvement [6-8]. Therefore, in this research, stability precision is always treated as the key performance index to evaluate the whole system.

Except for the casual environmental disturbances caused by carrier movements, winds, noises, etc., the over-all stability precision can also be severely affected by other errorcausing sources (ECS) related to the quality of the device itself, like assembly errors, mass eccentricities, and sensor measurement accuracies. For example, $\mathrm{Wu}$ et al. [9] studied the device stabilization with total warping variation model. Yu et al. [10] studied video stabilization based on the modeling of motion imaging. Mao et al. [11] proposed the continuous second-order sliding mode control based on disturbance observer for LOS stabilization. Chen et al. [12] studied the disturbance observer-based control and related methods. Dasgupta et al. [13] designed the disturbance observer for nonlinear systems by using a Hirschorn inverse approach. Gao et al. [14] carried out an analysis on the 


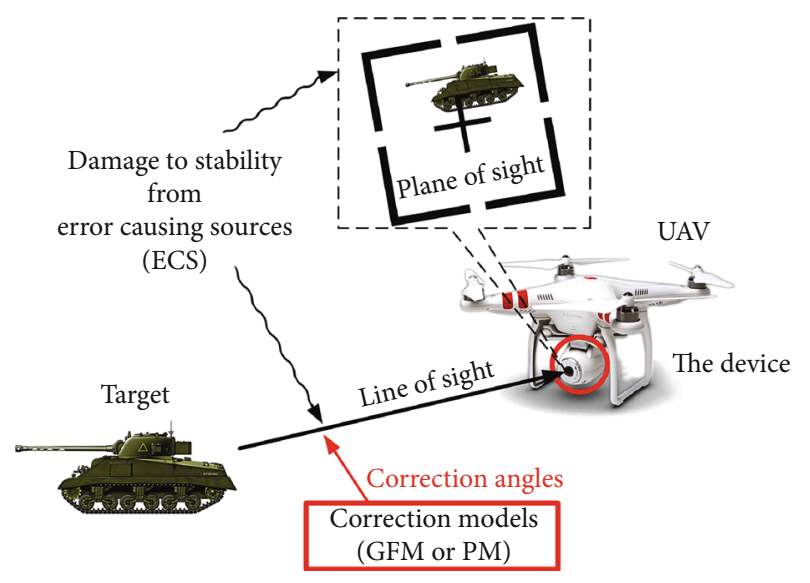

A better correction model will predict the deviations more accurately and finally achieve a higher stability precision.

FIgURE 1: Typical application of UAV photoelectric detection.

influence of assembly error of off-axis three-mirror optical system on imaging line-of-sight. Liu et al. [15] carried out a machining error analysis of freeform surface off-axis threemirror system based on optical performance evaluation.

However, these studies commonly adopt the methods of off-line modeling and precorrection before the real application. Comparatively, the real-time error correction is considered in this research, which is beneficial for the real-time improvement during the real application process.

The real-time compensation of the overall pointing errors will generally dissolve the disturbances coupled to the device base and improve the final precision. Specifically, to stabilize the line of sight (LOS) of the device, the kinematic pair of the azimuth frame and motor, and that of the pitch one, must be both used to compensate simultaneously. In this research, the two pairs are abbreviated as AZ and PI hereafter. However, the compensation performance will be greatly deteriorated by those ECS [16-18]. Therefore, during real applications, the extra error corrections aiming at dissolving the effects of ECS will be needed. The general correction process is shown in Figure 2.

The correction algorithm will be a parametric model used to predict the effects of ECS. It is used to calculate the pointing angle deviations between when those ECS exist and do not exist. Besides, when there are no ECS existing, the pointing angles are called theoretical pointing angles, which can be obtained by using analysis of general kinematics and compensation, and are often treated as the input parameters. In the real application scenarios, the real-time correction of those ECS effect will be realized by using extra servo control mechanism, including frames, motors, or more advanced structures, like multiaxis linkages, which are assembled on the outer side of AZ and PI. Those extra mechanisms will rotate according to the correction angles generated by using the algorithms above, so the deviations caused by ECS can be dissolved. Therefore, the design of the correction algorithm will be the key factor determining correction performance, which can be analyzed by comparing the final precision with the original precision without any correction. A better algorithm will predict the deviations more accurately and finally achieve a higher precision [19].

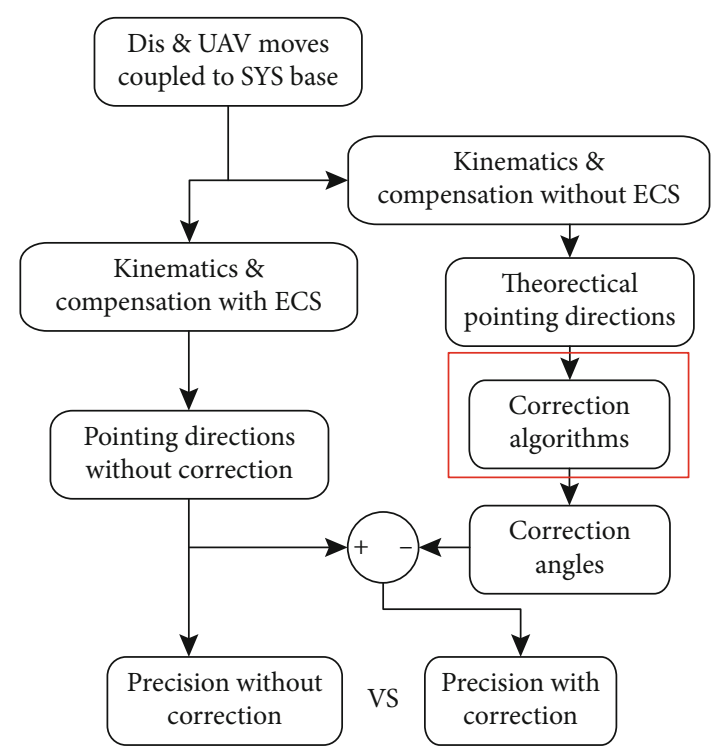

FIgURE 2: General error correction by using algorithms.

For instance, Wang et al. [20] proposed the real-time FPGA-based Kalman filter for constant and nonconstant velocity periodic error correction. Xingshun et al. [21] studied the real-time phase correction based on FPGA in the beam position and phase measurement system. Refan et al. [22] proposed the real-time global poisoning stability improvement by utilizing support vector machine. Sen et al. [23] studied the real-time correction of periodic nonlinearity in detection for scanning beam interference. Yu et al. [24] carried out research on dynamic tracking and compensation method for imaging, and there are other similar researches related to the error correction modeling [25-27]. There are plenty of researches concerning real-time error corrections using methods like collective morphing, PID algorithms, and lateral control [28-30]. However, these studies typically focused on only one kind of error like that of beam axis, sensors, motors, or accelerators, without comprehensive modeling and analysis. Besides, they barely focused on the two-axis stabilization mechanism, which is commonly adopted by UAV photoelectric detection.

In this research, based on our previous modeling works of the precision and various ECS, different correction algorithms are modeled and introduced into the overall simulation model, and the performances of those correction algorithms are compared. At last, the chosen algorism is validated by carrying out both simulation and experiment using data of a real product. Therefore, the real-time error correction of the UVA stability precision is comprehensively studied. Furthermore, it can be concluded that this research fills the technical deficiency of using a comprehensive method to compensate for the stability error of UAV photoelectric detection and provides a pioneering theoretical guidance for the related engineering applications in the future.

The organization of this paper is as follows: the background and literature review are presented in Section 1; the modeling and simulations of ECS are presented in Section 2; the error correction modeling is presented in Section 3; 
the simulation and validation experiments are presented in Section 4; the final conclusions and future work are presented in Section 5.

\section{Modeling and Simulations of UAV, Stability Precision, and Error-Causing Sources}

Based on our previous research of error modeling [31], the necessary predefinitions are shown as follows, and all modeling components are integrated together for the later analysis. Furthermore, because the device base is considered as being fixed to the UAV body, the dynamic modeling and related analysis of UAV can be simplified as that of the device base, which is presented as follows:

(i) The final precision is denoted as an index of RMS, and it is defined as the root mean square of the angular deviations $\Delta \theta_{s i}$. The values of $\Delta \theta_{s i}$ are the differences between the ideal pointing positions and the real ones, and they are also defined as the pointing errors. The specific method to calculate $\Delta \theta_{s i}$ and RMS can be seen in our previous work [31] by using a series of statistic indexes

(ii) The angular velocity of the device base related to the ground is denoted as $\vec{\omega}_{i b}=\left[\omega_{i b x}, \omega_{i b y}, \omega_{i b z}\right]^{T}$. That of AZ is denoted as $\vec{\omega}_{i a}=\left[\omega_{i a x}, \omega_{i a y}, \omega_{i a z}\right]^{T}$; the angle and the velocity of $\mathrm{AZ}$ compensation, which are driven by $\mathrm{AZ}$ motor, are denoted as $\theta_{a}$ and $\dot{\theta}_{a}$. Those parameters of PI are denoted as $\vec{\omega}_{i p}=$ $\left[\omega_{i p x}, \omega_{i p y}, \omega_{i p z}\right]^{T}, \theta_{p}$, and $\dot{\theta}_{p}$, respectively

(iii) For the ECS modeling, in this research, the commonly-seen ECS of perpendicularity deviations including $\vec{\delta}_{a}=\left[\delta_{a x}, \delta_{a y}, 0\right]^{T}$ and $\vec{\delta}_{p}=\left[\delta_{p x}, 0, \delta_{p z}\right]^{T}$, which can be measured by using high precision instruments [32], run-outs [33], including $\vec{\gamma}_{a}=$ $\left[\gamma_{a x}, \gamma_{a y}, 0\right]^{T}$ and $\vec{\gamma}_{p}=\left[\gamma_{p x}, 0, \gamma_{p z}\right]^{T}$, and assembly deviations of gyroscope locations [34] including $\vec{\varepsilon}_{a}$ $=\left[\varepsilon_{a x}, \varepsilon_{a y}, 0\right]^{T}$ and $\vec{\varepsilon}_{p}=\left[\varepsilon_{p x}, 0, \varepsilon_{p z}\right]^{T}$, are considered

(iv) For the kinematics modeling, as mentioned, AZ and PI must be both used to compensate simultaneously. According to kinematics analysis of homogeneous coordinate transformation put forward by [35], the rotation transformation matrix from the device base to $\mathrm{AZ}$ and that from $\mathrm{AZ}$ to PI is denoted as $R_{b}^{a}$ and $R_{a}^{p}$ . Those of perpendicularity deviations are denoted as $R_{a}^{\prime}$ and $R_{b}^{\prime}$, those of run-outs are denoted as $\Delta R_{a}$ and $\Delta R_{p}$, and those of gyroscope location deviations are denoted as $R_{a g}$ and $R_{p g}$

More details of those ECS and related kinematics analysis can be seen in our previous work [31]. By integrating all the above modules, the overall stability precision is modeled in Simulink, which is shown in Figure 3. The original system base angular velocity $\vec{\omega}_{i b}=\left[\omega_{i b x}, \omega_{i b y}, \omega_{i b z}\right]^{T}$ is set as the input. All of the modules of the kinematics, components, and ECS are embedded in the middle, and the details of those modules can also be seen in our previous work [31]. The final index of stability precision RMS is set as the output.

\section{Error Correction Modeling}

3.1. Necessary Predefinitions and Preparations before the Modeling. As mentioned, the error correction process is used to dissolve the effects caused by ECS. The correction algorithm model is used to predict the pointing angle deviations between when those ECS exist and do not exist. When there are no ECS existing, those are called the theoretical pointing angles. For the case of the two-axis-two-frame structure of $\mathrm{AZ}$ and PI, the theoretical pointing angles are denoted as [ $\alpha_{i}, \beta_{i}$ ], where $\alpha_{i}$ denotes the $\mathrm{AZ}$ pointing angle, $\beta_{i}$ denotes the PI one, $i=1,2,3, i^{-}, n_{m}$, and $n_{m}$ is the total number of the samples used for modeling. $\left[\alpha_{i}, \beta_{i}\right]$ can be obtained by using methods of general kinematics and compensation and are often treated as the input parameters of the correction model.

Respectively, when there are ECS existing, those are called the real pointing angles, which are denoted as $\left[\widehat{\alpha}_{i}, \widehat{\beta}_{i}\right]$ . The deviations between the theoretical and the real are denoted as $\left[\delta \alpha_{i}, \delta \beta_{i}\right]$. In that case, the general form of the error correction model can be expressed as (1), where $f\left(\alpha_{i}\right.$, $\left.\beta_{i}\right)$ and $g\left(\alpha_{i}, \beta_{i}\right)$ are the model functions, and $\varepsilon_{i}$ and $\eta_{i}$ are the prediction deviations of the model.

$$
\left\{\begin{array}{l}
\delta \alpha_{i}=\widehat{\alpha}_{i}-\alpha_{i}=f\left(\alpha_{i}, \beta_{i}\right)+\varepsilon_{i}, \\
\delta \beta_{i}=\widehat{\beta}_{i}-\beta i=g\left(\alpha_{i}, \beta_{i}\right)+\eta_{i} .
\end{array}\right.
$$

Therefore, the modeling process is to find the most appropriate forms of $f\left(\alpha_{i}, \beta_{i}\right)$ and $g\left(\alpha_{i}, \beta_{i}\right)$ to obtain the minimum accumulation values of $\varepsilon_{i}$ and $\eta_{i}$, which is expressed by (2):

$$
\left\{\begin{array}{l}
\min \left(\sum_{i=1}^{n_{m}} \varepsilon_{i}^{2}\right)=\min \left\{\sum_{i=1}^{n_{m}}\left[f\left(\alpha_{i}, \beta_{i}\right)-\delta \alpha_{i}\right]^{2}\right\}, \\
\min \left(\sum_{i=1}^{n_{m}} \eta_{i}^{2}\right)=\min \left\{\sum_{i=1}^{n_{m}}\left[g\left(\alpha_{i}, \beta_{i}\right)-\delta \beta_{i}\right]^{2}\right\} .
\end{array}\right.
$$

The $n_{m}$ groups of sampling values of $\left[\delta \alpha_{i}, \delta \beta_{i}\right]$ and $\left[\alpha_{i}, \beta_{i}\right]$ are the data basis for the modeling process. Based on simulation models presented in Section 2 and by carrying out the simulation with all those ECS set to zeros, the signal values of $\Delta \theta_{s z i}$ and $\Delta \theta_{s y i}$ are taken as the sample values of $\left[\alpha_{i}, \beta_{i}\right]$ by definition. Similarly, the differences of those signal values, between when those ECS are set to certain values and zeros, are taken as the sample values of $\left[\delta \alpha_{i}, \delta \beta_{i}\right]$. Additionally, the ECS are all set to be 0.5 degrees considering the actual manufacturing and assembly accuracy. The sampling data process is shown in Figure 4. 


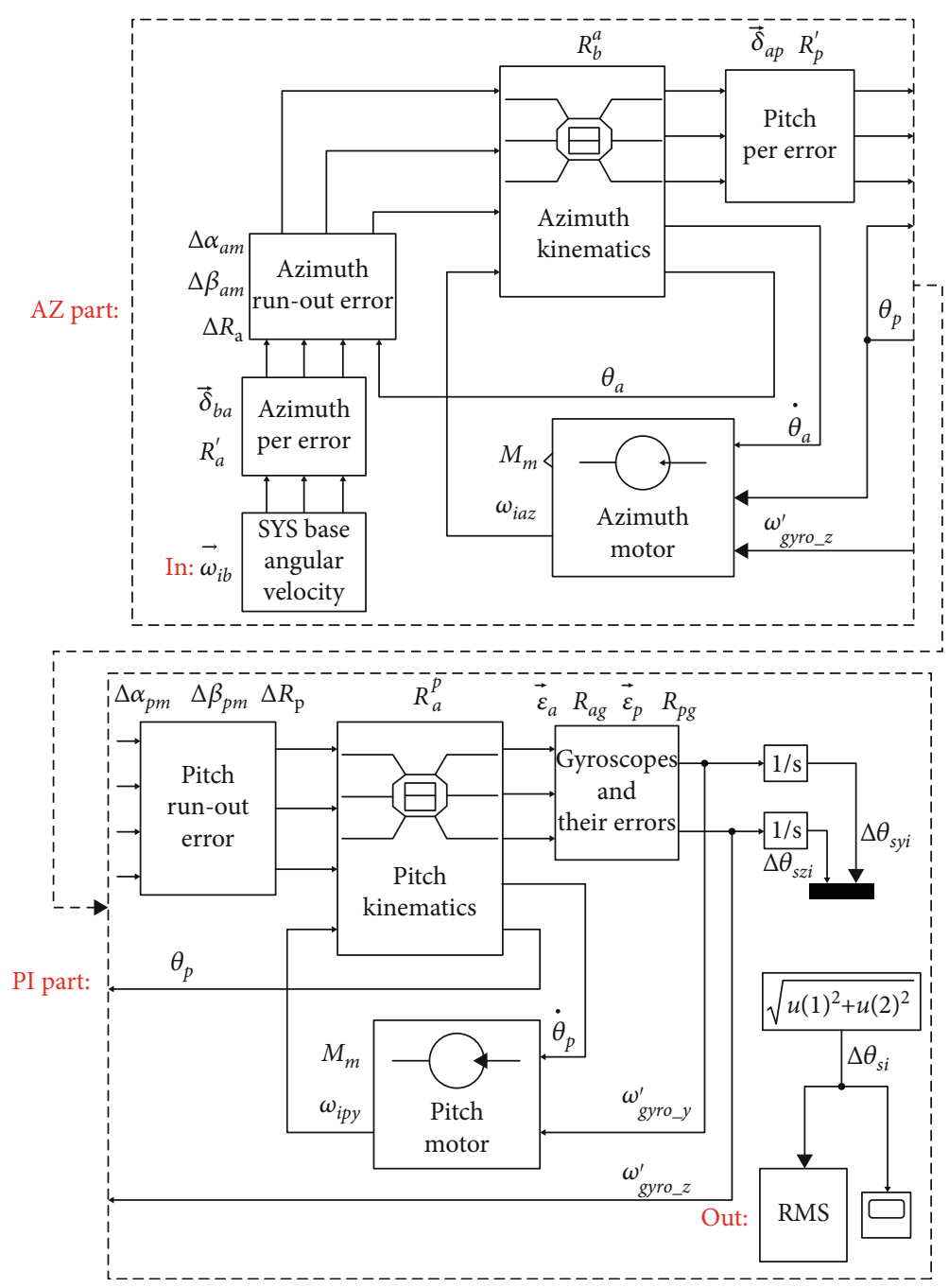

FIGURE 3: Overall simulation modeling of the stability precision.

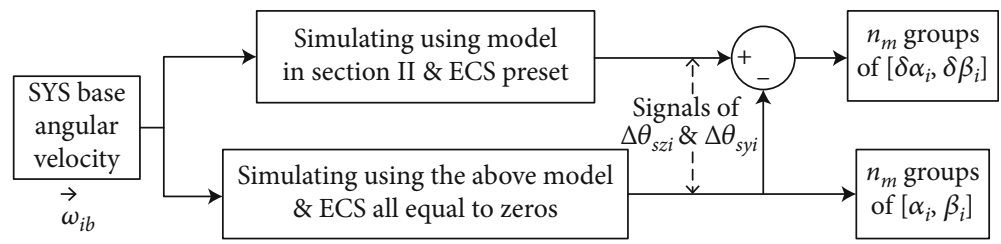

FIGURE 4: Data-acquiring of the samples used for the modeling.

About more details of the above data-acquiring process, the UAV movements, and other various disturbances coupled to the device base, namely, $\vec{\omega}_{i b}=\left[\omega_{i b x}, \omega_{i b y}, \omega_{i b z}\right]^{T}$, are simulated by using back-ward deduction of a power spectrum. In addition, based on real measurement data of UAV movements in outdoor environments, the power spectrum is obtained by using the method of correction function estimation [36]. The backward deduction means that the amplitude of the simulated signal is obtained by inverse Fourier transform of the power spectrum data, the phase is randomly generated, and the three directions of $\vec{\omega}_{i b}$ are all set equal.
Those processes and obtained data are shown in Figure 5, and the relative MATLAB codes are also attached as supplementary files.

Based on the above background, 13600 groups of $\left[\delta \alpha_{i}, \delta\right.$ $\left.\beta_{i}\right]$ and $\left[\alpha_{i}, \beta_{i}\right]$ are obtained, namely, $n_{m}$ is set as 13600 . Those obtained data groups are also attached as supplementary files, which can be used as the basis for the error correction modeling.

3.2. Global Function Model. Global function model (GFM) [37] takes the form of a spherical function of Fourier series 


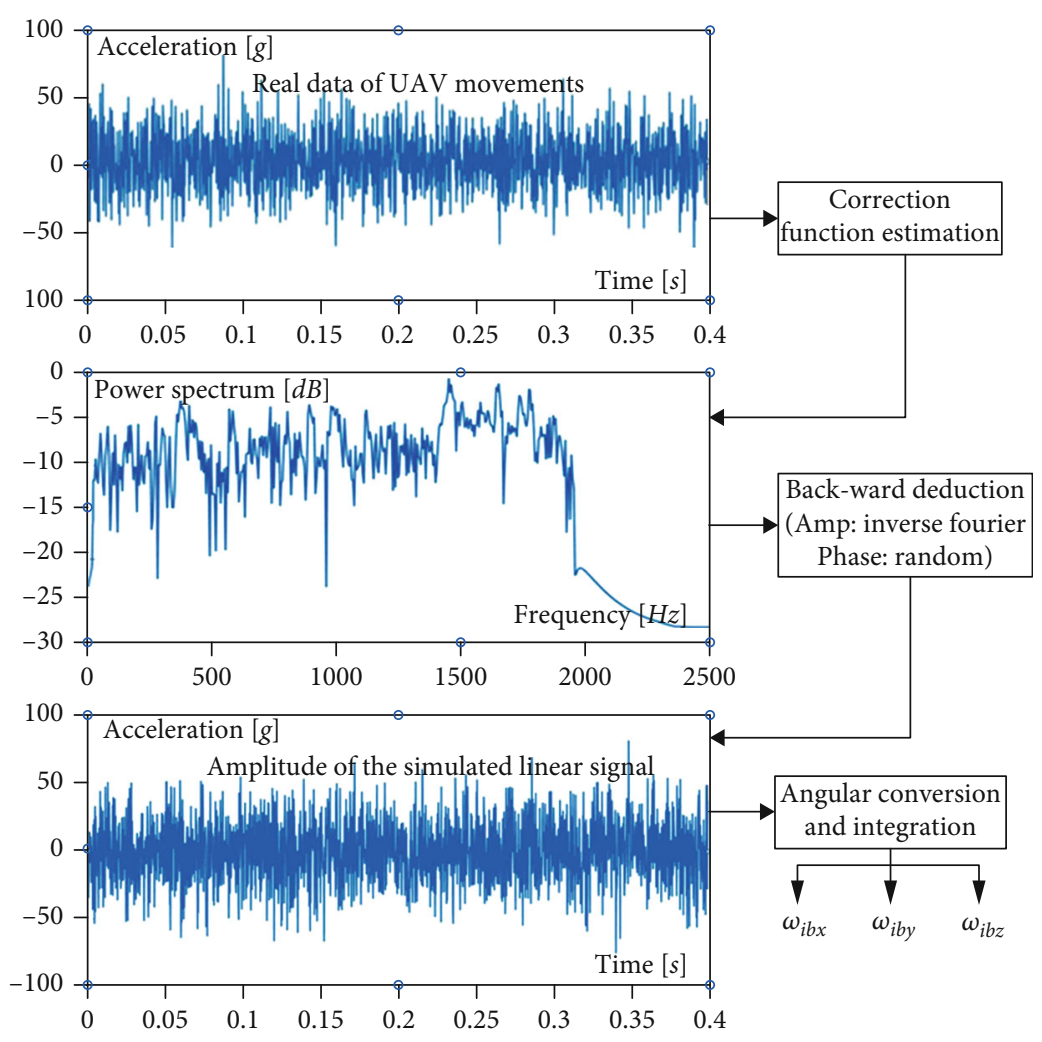

FIGURE 5: Simulation of the UAV moves and other disturbances.

expansion $h(\alpha, \beta)$ as (3), where $0 \leq \alpha \leq 2 \pi$ and $0 \leq \beta \leq 2 \pi$ denote the rotary angles of a time point, $A_{m}(\alpha)$ and $B_{m}(\alpha)$ denote the Fourier series coefficients, and $m$ denotes the Fourier series number of an expansion term.

$$
h(\alpha, \beta)=\sum_{m=0}^{\infty}\left[A_{m}(\alpha) c(m \beta)+B_{m}(\alpha) s(m \beta)\right] .
$$

Then by using the spherical function as the basis, $A_{m}(\alpha)$ and $B_{m}(\alpha)$ are expanded in $[0,2 \pi]$ as $(4)$, where $P_{l}^{m}(c \alpha)$ is the $l$-order associated Legendre polynomial [38], and $A_{l}^{m}$ and $B_{l}^{m}$ are the coefficients.

$$
\begin{aligned}
& A_{m}(\alpha)=\sum_{l=m}^{\infty} A_{l}^{m} P_{l}^{m}(c \alpha), \\
& B_{m}(\alpha)=\sum_{l=m}^{\infty} B_{l}^{m} P_{l}^{m}(c \alpha) .
\end{aligned}
$$

By introducing (4) into (3), we get (5), which can be further expanded as a form of polynomial shown as (6).

$$
h=\sum_{m=0}^{\infty} \sum_{l=m}^{\infty}\left[A_{l}^{m} c(m \beta)+B_{l}^{m} s(m \beta)\right] P_{l}^{m}(c \alpha),
$$

$$
\begin{gathered}
h(\alpha, \beta)=a_{0}+a_{1} s \beta+a_{2} c \alpha c \beta+a_{3} s \alpha c \beta+a_{4} s^{2} \beta+ \\
a_{5} c \alpha s \beta c \beta+a_{6} s \alpha s \beta c \beta+a_{7} c 2 \alpha c^{2} \beta+a_{8} s 2 \alpha c^{2} \beta+ \\
a_{9} s^{3} \beta+a_{10} c \alpha s^{2} \beta c \beta+a_{11} s \alpha s^{2} \beta c \beta+a_{12} c 2 \alpha c^{2} \beta s \beta .
\end{gathered}
$$

Therefore, according to the definition of error correction models shown in (1), the GFM model can be expressed as (7) by introducing (6) into (1) for the AZ and PI, respectively:

$$
\left\{\begin{array}{l}
\delta \alpha_{i} c \beta_{i}=f\left(\alpha_{i}, \beta_{i}\right)= \\
a_{0}+a_{1} s \beta_{i}+\cdots+a_{12} c 2 \alpha_{i} c^{2} \beta_{i} s \beta_{i}+\varepsilon_{i}, \\
\delta \beta_{i}=g\left(\alpha_{i}, \beta_{i}\right)= \\
b_{0}+b_{1} s \beta_{i}+\cdots+b_{12} c 2 \alpha_{i} c^{2} \beta_{i} s \beta_{i}+\eta_{i} .
\end{array}\right.
$$

Equation (7) is expressed in form of a matrix as in (8):

$$
\left[\begin{array}{l}
\delta \alpha \\
\delta \beta
\end{array}\right]=\left[\begin{array}{l}
A_{\alpha} \\
A_{\beta}
\end{array}\right] x+\left[\begin{array}{l}
\varepsilon \\
\eta
\end{array}\right] .
$$

Those symbols in (8) are explained in (9). By carrying out regression analysis using the 13600 data groups mentioned in Subsection 3.1, we get the result of $x=[-5.495$ $\times 10^{3}, 1.000 \times 10^{-5}, 1.000 \times 10^{-6}, 1.000 \times 10^{-6},-1.211 \times 10^{2}$ 


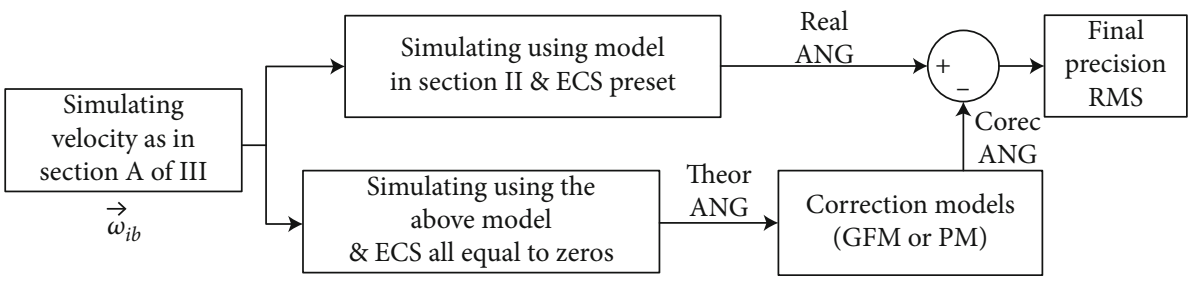

Figure 6: The simulation process of error correction.

$, 1.000 \times 10^{-5}, 1.906 \times 10^{4}, 5.495 \times 10^{3},-4.239 \times 10^{-1}, 3.617$ $\times 10^{-1}, 1.098 \times 10^{0}, 1.000 \times 10^{-6}, 1.000 \times 10^{-5},-1.476 \times 10^{0}$ $, 2.913 \times 10^{4}, 3.045 \times 10^{3},-1.052 \times 10^{-1}, 1.000 \times 10^{-6}, 1.000$ $\left.\times 10^{-4}, 1.000 \times 10^{-5}, 1.000 \times 10^{-6}\right]$, which means that the GFM model is obtained. Apparently, GFM takes the form originated from general spherical function, and it contains many parameters without specific physical meaning. That is not beneficial for deep understanding of ECS.

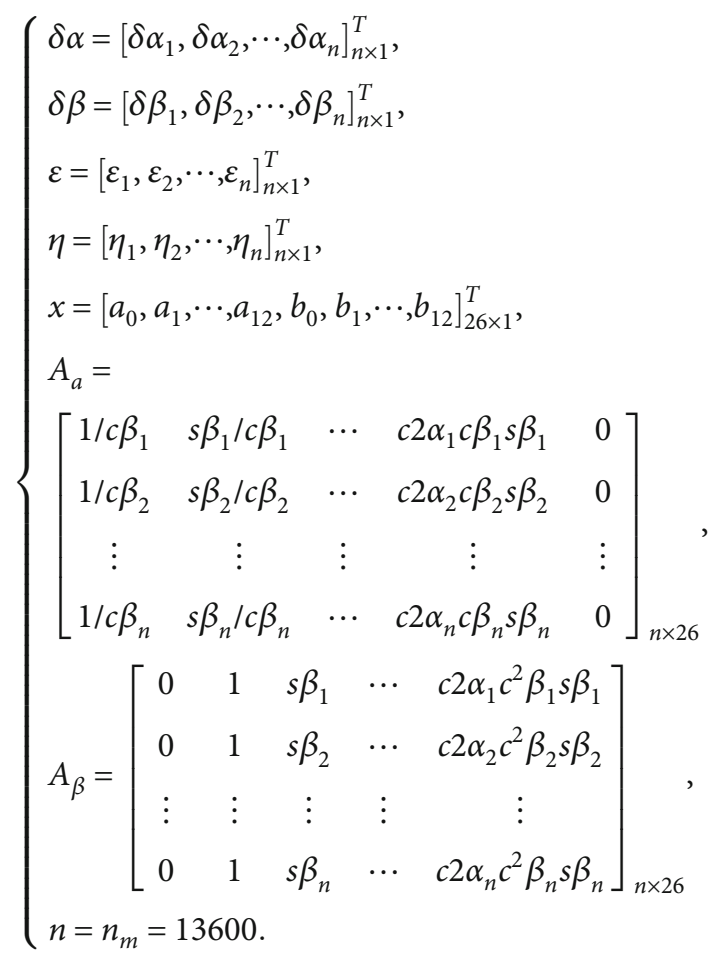

3.3. Parametric Model. Parametric model (PM) is established by using general movement theory and previous analysis results of ECS in Section 2. The target position in the base coordinate $C(b)$ can be calculated by (10), where $P_{p g i}$ is one of the sample values of the target position in the $C(p g)$ coordinate at the end of the coordinate series, and $P_{b i}=\left[x_{b i}, y_{b i}, z_{b i}\right]$ is that in the $C(b)$ coordinate at the begging of the series. By doing that, the real-time target position in the absolute coordinate can be obtained just by using the gyroscope signals.

$$
P_{b i}=R_{a}^{\prime} \Delta R_{a} R_{b}^{a} R_{p}^{\prime} \Delta R_{p} R_{a}^{p} R_{a g} R_{p g} P_{p g i} .
$$

TABle 1: Preset parameters of the simulation.

\begin{tabular}{lc}
\hline Software version & MATLAB R2019b Simulink \\
\hline Duration time & $0-100 \mathrm{~s}$ \\
Solver type & Variable-step (ode45) \\
Tolerance of relative and absolute & Automatic \\
Output option & Refine output \\
\hline
\end{tabular}

We introduce the contents in Section 2 into (10) and then simplify the result by adhering to rules as follows: first, any of the elements $\Delta$ belongs to ECS are treated as small angles, so $s \Delta \approx 0$, and $c \Delta \approx 1$; second, all of the high-order elements are neglected. Simultaneously, the transfer relation between the rectangular coordinate and spherical coordinate is shown as (11), where $d$ is the distance away from the target.

$$
\left\{\begin{array} { l } 
{ \operatorname { t a n } ( \alpha _ { i } + \delta \alpha _ { i } ) = - \frac { y _ { b i } } { x _ { b i } } } \\
{ \operatorname { t a n } ( \beta _ { i } + \delta \beta _ { i } ) = \frac { z _ { b i } } { d } }
\end{array} \Rightarrow \left\{\begin{array}{l}
\frac{s \alpha_{i}+c \alpha_{i} \delta \alpha_{i}}{c \alpha_{i}-s \alpha_{i} \delta \alpha_{i}}=-\frac{y_{b i}}{x_{b i}} \\
s \beta_{i}+c \beta_{i} \delta \beta_{i}=\frac{z_{b i}}{d}
\end{array}\right.\right.
$$

Finally, the PM model can be expressed as (12):

$$
\left\{\begin{array}{l}
\delta \alpha_{i} \approx\left(\varepsilon_{a y}+\varepsilon_{a x}+\delta_{p x}\right)-\left(\delta_{p z}+\gamma_{a y}\right) \tan \beta_{i} \\
+\gamma_{p x} \sec \beta_{i}+\delta_{a x} s \alpha_{i} \tan \beta_{i}-\delta_{a y} c \alpha_{i} \tan \beta_{i}+\varepsilon_{i} \\
\delta \beta_{i} \approx\left(\varepsilon_{p z}+\varepsilon_{p x}+\gamma_{a x}\right)+\delta_{a x} c \alpha_{i}+\delta_{a y} s \alpha_{i}+\eta_{i}
\end{array}\right.
$$

Equation (12) is expressed in form of a matrix as (13):

$$
\left[\begin{array}{l}
\delta \alpha \\
\delta \beta
\end{array}\right]=\left[\begin{array}{l}
A_{\alpha} \\
A_{\beta}
\end{array}\right] x+\left[\begin{array}{l}
\varepsilon \\
\eta
\end{array}\right] .
$$

Those symbols in Eq. (13) are explained in Eq. (14), where $x_{1}=\varepsilon_{a y}+\varepsilon_{a x}+\delta_{p x}, x_{2}=-\left(\delta_{p z}+\gamma_{a y}\right), x_{3}=\gamma_{p x}, x_{4}=$ 
TABLE 2: Necessary parameters of the product for the experiment.

\begin{tabular}{|c|c|c|c|}
\hline PARM & Descriptions & Values & Units \\
\hline$J_{m a}$ & Rotary inertia of the azimuth motor & 0.02 & $\mathrm{~kg} \cdot \mathrm{m}^{2}$ \\
\hline$J_{L a}$ & Rotary inertia of the azimuth load & 0.04 & $\mathrm{~kg} \cdot \mathrm{m}^{2}$ \\
\hline$J_{m p}$ & Rotary inertia of the pitch motor & 0.01 & $\mathrm{~kg} \cdot \mathrm{m}^{2}$ \\
\hline$J_{L p}$ & Rotary inertia of the pitch load & 0.02 & $\mathrm{~kg} \cdot \mathrm{m}^{2}$ \\
\hline$R_{m}$ & Total resistor of the two armature circuits & 8.60 & $\Omega$ \\
\hline$L_{m}$ & Total inductance of the armature circuits & 0.01 & $H$ \\
\hline$D_{L}$ & Damping coefficient of the two motors & 10 & I \\
\hline$K_{L}$ & Elastic coefficient of the two motors & 100000 & l \\
\hline$C_{e}$ & EMF coefficient of the two motors & 0.33 & I \\
\hline$C_{m}$ & Motor torque coefficient of the two motors & 0.33 & l \\
\hline$K_{p}$ & Amplification coefficient of driving circuit & 20 & l \\
\hline$R_{s}$ & Resistor of the driving circuit & 0.4 & $\Omega$ \\
\hline$K_{g}$ & Amplification coefficient of the gyroscopes & $15 / \pi$ & l \\
\hline$\omega_{g}$ & Natural frequency of the gyroscopes & $200 \pi$ & $\mathrm{rad} / \mathrm{s}$ \\
\hline$\lambda_{g}$ & Damping coefficient of the gyroscopes & 0.7 & I \\
\hline
\end{tabular}

$\delta_{a x}, x_{5}=-\delta_{a y}, y_{1}=\varepsilon_{p z}+\varepsilon_{p x}+\gamma_{a x}, y_{2}=\delta_{a x}$ and $y_{3}=\delta_{a y}$.

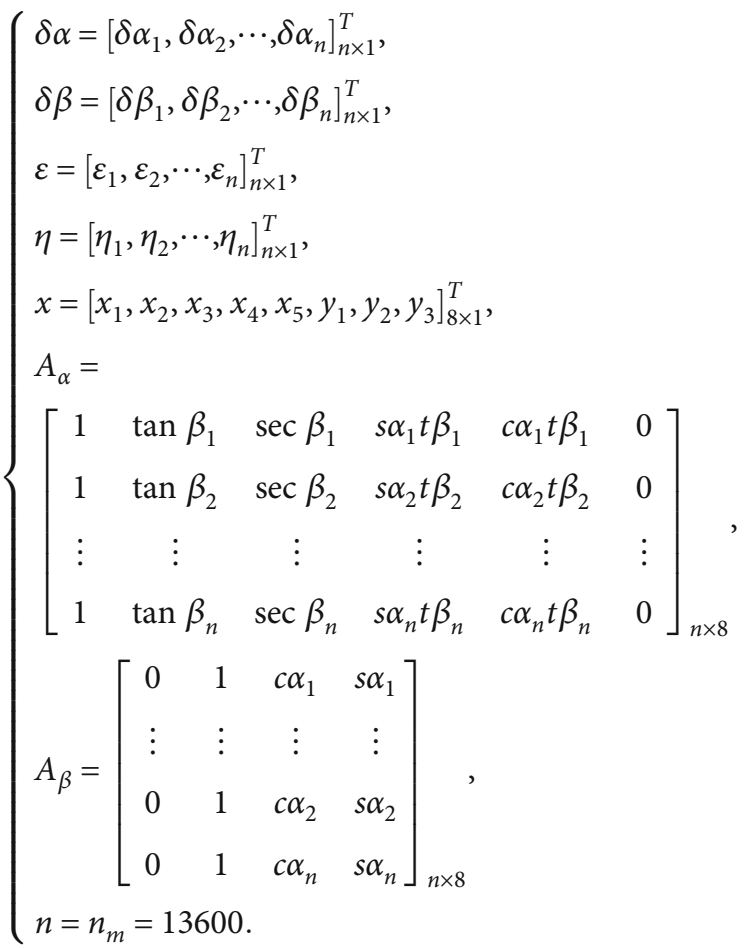

By carrying out regression analysis using the 13600 data groups mentioned in Subsection 3.1, we get the result of $x=\left[-5.963 \times 10^{3}, 3.571 \times 10^{-1}, 5.963 \times 10^{3},-9.279 \times 10^{2}\right.$, $\left.1.000 \times 10^{-5}, 7.977 \times 10^{3},-7.977 \times 10^{3}, 9.472 \times 10^{-1}\right]$, which means that the PM model is obtained. Compared to
TABLE 3: The simulation results of error correction.

\begin{tabular}{lcc}
\hline $\begin{array}{l}\text { Correction model } \\
\text { used }\end{array}$ & $\begin{array}{c}\text { Stability } \\
\text { precision RMS } \\
(\mu \mathrm{rad})\end{array}$ & $\begin{array}{c}\text { Improvement percentage } \\
\text { related to the original (\%) }\end{array}$ \\
\hline $\begin{array}{l}\text { The original RMS } \\
\text { without correction }\end{array}$ & 169.388 & 0 \\
GFM & 165.703 & 2.175 \\
PM & 135.207 & 20.179 \\
ADRC & 156.232 & 7.766 \\
DAC & 163.432 & 3.516 \\
CHADC & 149.523 & 11.728 \\
\hline
\end{tabular}

GFM, PM has few parameters. Those parameters can be explained by referring to the meaning of the predefined ECS, which is beneficial for possible future improvement.

\section{Simulation and Validation Experiment of Error Correction}

Based on the modeling results in Section 3, both simulation and validation experiments are carried out to evaluate the performances of the modeling methods.

4.1. Simulations of Error Correction. The simulation process is shown in Figure 6, in which the device base movements are simulated by signals generated in Subsection 3.1. The overall integrated precision model in Section 2 is used to generate real-time real pointing angles and theoretical pointing angles. Different correction models, including GFM and PM in Subsections 3.2 and 3.3, and the most commonly used models of ADRC, DAC, and CHADC in [4, 12, 20], are all used to calculate the correction angles. The final stability 

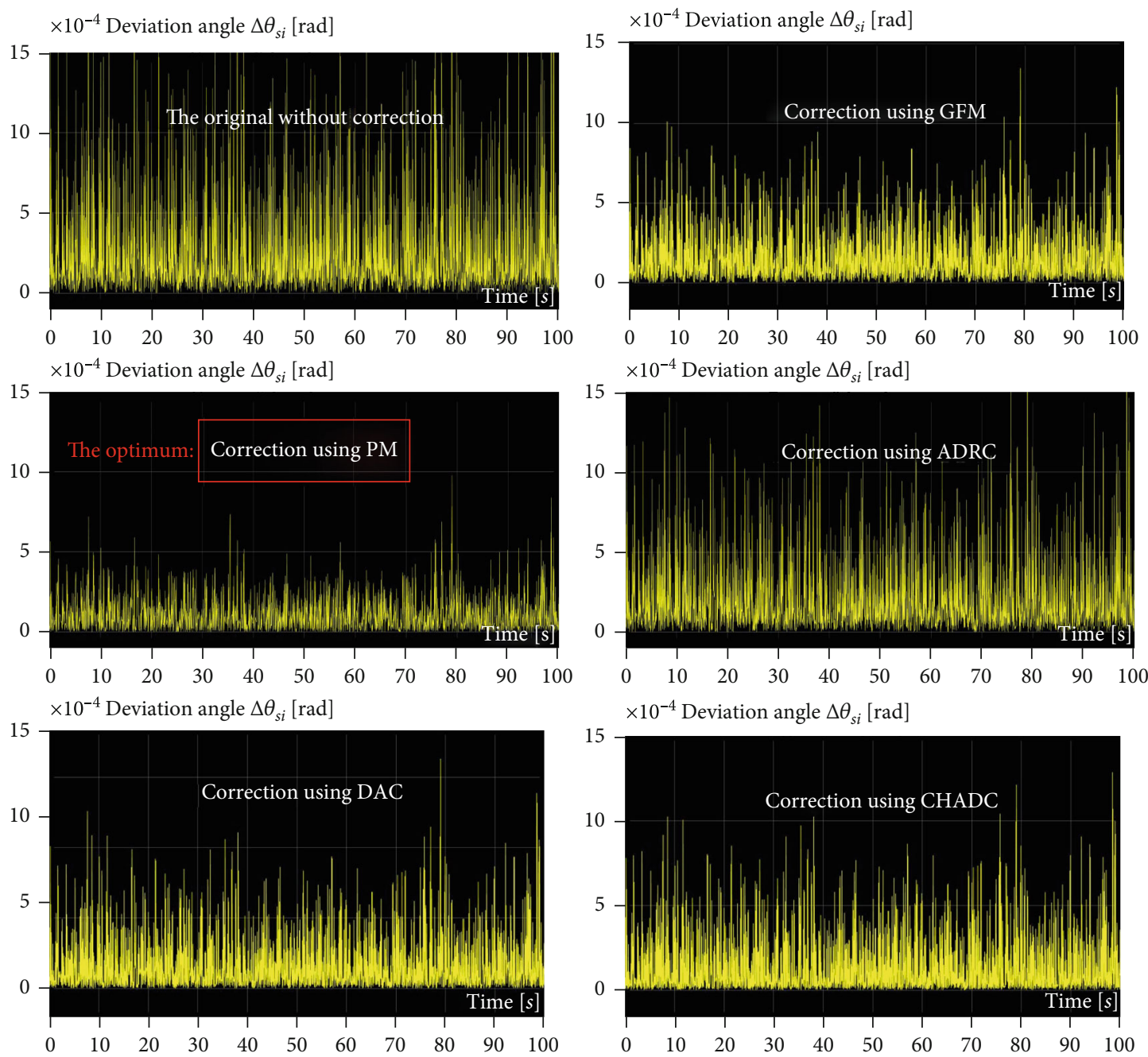

Figure 7: The deviations $\Delta \theta_{s i}$ along the simulations.

precisions after correction are also compared to evaluate the performances of the correction models.

The modeling and simulations are all carried out in MATLAB R2019b Simulink, and the MATLAB codes of the simulation works are available online at Supplementary Materials.

The necessary parameters of the simulation are listed in Table 1, and other parameters for the calculations are set equal to those listed in Table 2 shown later, which is originated from a real pod product. The simulation results are listed in Table 3. The deviation angles $\Delta \theta_{s i}$ along the simulation process are presented in Figure 7, which is used to calculate the final RMS.

From Table 3 and Figure 7, it is obvious that PM outperformed GFM and the others in improving the overall stability precision, and the improvement percentage can reach up to over $20 \%$.

4.2. Validation Experiment of Error Correction. For further validation of the correction models, validation experiments using a real product assembled on an experiment platform is carried out, as shown in Figure 8.
The details of the experiment platform are listed as follows:

(i) The high precision servo turntables is used to simulate the UAV movements and other disturbances coupled to the product base, namely, $\vec{\omega}_{i b}=$ $\left[\omega_{i b x}, \omega_{i b y}, \omega_{i b z}\right]^{T}$. Those movements are also generated by using signals in Subsection 3.1, and based on which, the theoretical pointing angles $\left[\alpha_{i}, \beta_{i}\right]$ are calculated in the background software to support the real-time error correction process using GFM or PM. Although all the simulation and experiments are carried out indoors, the movements are simulated according to the real outdoor environments

(ii) The photoelectric system product is a UAV detection pod

(iii) The PSD position measurement system is used to measure the real pointing angles $\left[\widehat{\alpha}_{i}, \widehat{\beta}_{i}\right]$ and further calculate the final stability precision RMS 


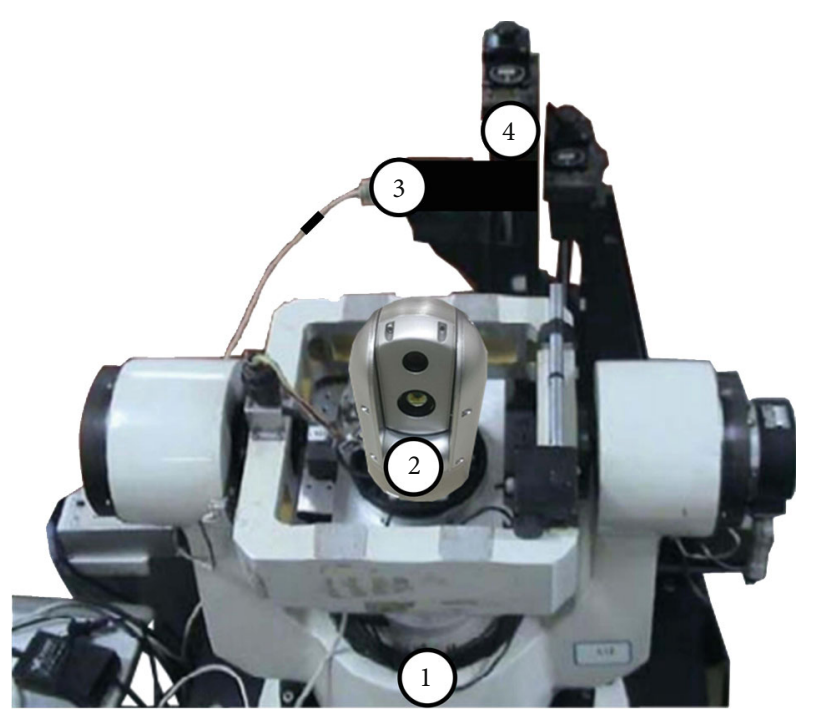

FIGURE 8: The studied product on the experiment platform. (1) High precision servo turntable. (2) Photoelectric system product. (3) PSD position measurement system. (4) Two-axis position platform.

TABLE 4: The validation experiment results of error correction.

\begin{tabular}{lcc}
\hline $\begin{array}{l}\text { Correction model } \\
\text { used }\end{array}$ & $\begin{array}{c}\text { Stability } \\
\text { precision RMS } \\
(\mu \mathrm{rad})\end{array}$ & $\begin{array}{c}\text { Improvement percentage } \\
\text { related to the original }(\%)\end{array}$ \\
\hline $\begin{array}{l}\text { The original RMS } \\
\text { without correction }\end{array}$ & 357.453 & 0 \\
GFM & 343.214 & 3.983 \\
PM & 302.382 & 15.407 \\
ADRC & 347.453 & 2.798 \\
DAC & 332.675 & 6.932 \\
CHADC & 329.765 & 7.746 \\
\hline
\end{tabular}

(iv) The two-axis position platform is used to adjust the position of the PSD system for better measurement

By referring to real measurement data of the pod product, the necessary parameters, used for the theoretical pointing angles calculation, are preset before the experiments. Those parameters are listed in Table 2.

GFM, PM, and other commonly used methods are both used to do the correction. The experiment results are listed in Table 4. From Table 4, PM also outperformed GFM and the others, and the improvement percentage can reach up to over $15 \%$.

\section{Conclusions and Future Work}

The major contributions of this research are as follows. Additionally, the nomenclature is presented in the end.

(1) Stability precision error correction of UAV photoelectric detection is comprehensively defined and analyzed. The analysis results and related data can be valuable guidance for real application
(2) Error correction models including global function model GFM and parametric model PM are established. The models are validated by carrying out both simulations and validation experiments and can be used in real applications

(3) The performances of the studied error correction models are compared and analyzed. PM outperformed GFM and other methods comprehensively, and the improvement percentage can reach up to over $20 \%$ for the simulation, and over $15 \%$ for the experiment

The limits of this research are listed as follows: (1) So far, only linear models are studied. Other error correction models with nonlinear expressions or fuzzy algorithms should be considered. (2) Other error sources and situations except for assembly errors to further verify the proposed analysis method should be considered, like mass eccentricities, deviations gyro measurements, and various kinds of noises. (3) The limitations, constraints used in this research, and the possibility of uncertainty are not concerned, like the changes of error distributions, target locations, and UAV linear disturbances.

As the next step, we will investigate other error correction methods like semiparametric model, BP neural net, and machining learning. The same is true for other error-causing sources and situations, like frictions, structural eccentricity, and inertia coupling. The definition of limitations and constraints should be well-adjusted according to the scenario. For example, the linear disturbances of UAV and target movements may have a great influence on the results. Similar control mechanism of more complex system with more dimensions or new materials will be studied, like two-axisfour-frame and three-axis-four-frame used on larger carriers.

\section{Nomenclature}

UAV: Unmanned aerial vehicle

LOS: $\quad$ Line of sight

AZ: $\quad$ Kinematic pair of the azimuth frame and motor

PI: $\quad$ Kinematic pair of the pitch frame and motor

ECS: $\quad$ Error causing sources

RMS: $\quad$ System precision; root mean square of the angular deviation between the ideal and the real pointing positions

$t_{s}, T_{s}, n_{s}: \quad$ Total sampling time in $[s]$, sampling period in $[s$ ], sampling number of system accuracy

$\Delta \bar{\theta}: \quad$ Mean of sampling values

$\Delta \theta_{s i}: \quad$ Sample value of the $i$ th sample and its components

$\sigma_{\Delta \theta}: \quad$ Standard error of sample values

$\vec{\omega}_{i b}$ : Angular velocity vector of the system base in the inertial coordinated in $[\mathrm{rad} / \mathrm{s}]$

$\vec{\omega}_{i a}$ : Angular velocity vector of the azimuth in the inertial coordinate $[\mathrm{rad} / \mathrm{s}]$

$\theta_{a}, \dot{\theta}_{a}: \quad$ Rotation angle in $[\mathrm{rad}]$ and angular velocity in $[\mathrm{rad} / \mathrm{s}]$ of azimuth compensation

$\vec{\omega}_{i p}$ : Angular velocity vector of the pitch in the inertial coordinate in $[\mathrm{rad} / \mathrm{s}]$ 
$\theta_{p}, \dot{\theta}_{p}: \quad$ Rotation angle in $[\mathrm{rad}]$ and angular velocity in $[\mathrm{rad} / \mathrm{s}]$ of pitch compensation

$R_{b}^{a}$ : $\quad$ Rotation transformation matrix from the system base coordinate to that of the azimuth

$R_{a}^{p}: \quad \quad$ Rotation transformation matrix from the azimuth coordinate to that of the pitch

$\vec{\delta}_{a}: \quad$ Perpendicularity deviation vector of azimuth axis assembling in the system base coordinate in $[\mathrm{rad}]$

$\vec{\delta}_{p}: \quad \quad \quad$ Perpendicularity deviation vector of pitch axis assembling in the azimuth coordinate in $[\mathrm{rad}]$

$R_{a}^{\prime}, R_{p}^{\prime}: \quad$ Rotation transformation matrixes affected by errors of axis perpendicularity of the azimuth and the pitch

$\vec{\gamma}_{a}, \gamma_{a x}, \gamma_{a y}$ : Run-outs vector of the azimuth, and its decomposed components of the maximum tilting oscillations around the $x$ and $y$ axes of the system base coordinate in [rad]

$\vec{\gamma}_{p}, \gamma_{p x}, \gamma_{p z}:$ Run-outs vector of the pitch, and its decomposed components of the maximum tilting oscillations around the $\mathrm{x}$ and $\mathrm{z}$ axes of the azimuth coordinate in [rad]

$\Delta R_{a}, \Delta R_{p}: \quad$ Rotation transformation matrixes affected by errors of run-outs of the azimuth and the pitch

$\vec{\varepsilon}_{a}, \varepsilon_{a x}, \varepsilon_{a y}$ : Vector of the azimuth gyroscope assembling deviation in the pitch coordinate, and its components around the $x$ and $y$ axes of the coordinate in $[\mathrm{rad}]$

$\vec{\varepsilon}_{p}, \varepsilon_{p x}, \varepsilon_{p z}:$ Vector of the pitch gyroscope assembling deviation in the pitch coordinate, and its components around the $x$ and $z$ axes of the coordinate in $[\mathrm{rad}]$

$R_{a g}, R_{p g}: \quad$ Rotation transformation matrixes affected by assembling errors of the gyroscope locations

$n_{m}: \quad$ Total number of the samples used for modeling

$\widehat{\alpha}_{i}, \widehat{\beta}_{i}: \quad$ Real pointing angles

$\delta \alpha_{i}, \delta \beta_{i}$ : Deviations between the theoretical and the real

$\varepsilon_{i}, \eta_{i}$ :

$A_{m}, B_{m}:$

$P_{l}^{m}(c \alpha)$ :

$P_{p g i}$ :

$P_{b i}:$

$\widehat{\alpha}_{i}, \widehat{\beta}_{i}$ :

$J_{m a}:$

$J_{L a}:$

$J_{m p}:$

$J_{L p}$ :

$R_{m}:$

$L_{m}:$

$D_{L}$ :

$K_{L}:$

$C_{e}:$

$C_{m}:$

$K_{p}:$

$R_{s}:$
$K_{g}:$

$\omega_{g}:$

$\lambda_{g}:$

Amplification coefficient of the gyroscopes

Natural frequency of the gyroscopes in $[\mathrm{rad} / \mathrm{s}]$

Damping coefficient of the gyroscopes.

\section{Data Availability}

The MATLAB codes of power correlation function and backward deduction mentioned above are included within the supplementary files. The figures used to show the data example for the modeling process, and the error correction process are also included within the supplementary files.

\section{Conflicts of Interest}

The authors declare that they have no conflicts of interest.

\section{Acknowledgments}

The authors would like to convey their sincere thanks to Mr. Haipeng $\mathrm{Gu}$ and $\mathrm{Mr}$. Zenan Xu from the School of Intelligence and Technology, National University of Defense Technology, and all the anonymous reviewers for their helpful suggestions on the quality improvement of our paper. This work was supported in part by the 66th batch of general grants from the China Postdoctoral Science Foundation of the Military System under Grant No. 2019M664022.

\section{Supplementary Materials}

Supplementary 1. Codes of power by correlation fun.

Supplementary 2. Correction processes.

Supplementary 3. Codes of backward deduction.

Supplementary 4. Data example.

\section{References}

[1] J. Qiu, D. Grace, G. Ding, J. Yao, and Q. Wu, "Blockchainbased secure spectrum trading for unmanned-aerial-vehicleassisted cellular networks: an operator's perspective," IEEE Internet of Things Journal, vol. 7, no. 1, pp. 451-466, 2020.

[2] J. Qiu, D. Grace, G. Ding, M. D. Zakaria, and Q. Wu, “Airground heterogeneous networks for $5 \mathrm{G}$ and beyond via integrating high and low altitude platforms," IEEE Wireless Communications, vol. 26, no. 6, pp. 140-148, 2019.

[3] L. Zhuchong, L. Kun, and Z. Wei, "Inertially stabilized platform for airborne remote sensing using magnetic bearings," IEEE/ASME Transactions on Mechatronics, vol. 21, no. 1, pp. 288-301, 2016.

[4] X. Zhou, B. Zhao, and H. Zhang, "Design of mechatronic system for a two-axis inertially stabilized platform in an APLI system," in Presented at Proceedings of the 2016 International Conference on Education, Management, Computer and Society, Shenyang, China, 2016https://www.atlantis-press.com/ proceedings/emcs-16/25848826.

[5] F. Dong, X. Lei, and W. Chou, "A dynamic model and control method for a two-axis inertially stabilized platform," IEEE Transactions on Industrial Electronics, vol. 64, no. 1, pp. 432439, 2017. 
[6] H. Li and S. Chen, "Detection ability mathematical model and performance evaluation method in visible-light photoelectric detection system," IEEE Sensors Journal, vol. 17, no. 6, pp. 1649-1655, 2017.

[7] H. Li and W. Gao, "Detection sensitivity calculation model and photoelectric detection performance analysis on laser light screens," IEEE Sensors Journal, vol. 16, no. 11, pp. 42584264, 2016.

[8] X. Liang, J. Zhang, L. Zhuo, Y. Li, and Q. Tian, "Small object detection in unmanned aerial vehicle images using feature fusion and scaling-based single shot detector with spatial context analysis," IEEE Transactions on Circuits and Systems for Video Technology, vol. 30, no. 6, pp. 1758-1770, 2020.

[9] H. Wu, L. Xiao, H. J. Shim, and S. Tang, "Video stabilisation with total warping variation model," IET Image Processing, vol. 11, no. 7, pp. 465-474, 2017.

[10] J. Yu, K. Xiang, X. Wang, S. Cao, and Y. Zhang, "Video stabilisation based on modelling of motion imaging," IET Image Processing, vol. 10, no. 3, pp. 177-188, 2016.

[11] J. Mao, S. Li, Q. Li, and J. Yang, "Continuous second-order sliding mode control based on disturbance observer for LOS stabilized system," in 2016 14th International Workshop on Variable Structure Systems (VSS), pp. 394-399, Nanjing, China, 2016.

[12] W.-H. Chen, J. Yang, L. Guo, and S. Li, "Disturbanceobserver-based control and related methods-an overview," IEEE Transactions on Industrial Electronics, vol. 63, no. 2, pp. 1083-1095, 2016.

[13] S. Dasgupta, S. Sadhu, and T. K. Ghoshal, "Designing disturbance observer for non-linear systems - a Hirschorn inverse approach," IET Science, Measurement and Technology, vol. 11, no. 2, pp. 164-170, 2017.

[14] L. Gao, X. Li, Z. Su et al., "Analysis on influence of installation error of off-axis three-mirror optical system on imaging line-of-sight," in Presented at 2017 International Conference on Optical Instruments and Technology: Optoelectronic Measurement Technology and Systems, Beijing, China, 2017https://ui.adsabs.harvard.edu/abs/2018SPIE10621E..1IG/ abstract.

[15] X. Liu, X. Zhang, H. Xu, and Z. Zhen, "Machining error analysis of freeform surface off-axis three-mirror system based on optical performance evaluation," Acta Optica Sinica, vol. 37, no. 8, pp. 0812005.1-0812005.9, 2017.

[16] D. Rajesh, A. V. Praveen, and M. R. Pasumarthi, "Design and analysis of two-axis seeker stabilization system," in Proceedings of the International Conference on Modern Research in Aerospace Engineering, pp. 97-107, Singapore, 2018.

[17] D. Rajesh, A. V. Praveen, and M. R. Pasumarthi, "Design and analysis of two-axis seeker stabilization system," in Presented at Proceedings of the International Conference on Modern Research in Aerospace Engineering, Singapore, 2018https:// link.springer.com/chapter/10.1007/978-981-10-5849-311citeas.

[18] V. M. Borodin, I. O. Spiridonov, and R. N. Faizutdinov, "Analysis of dynamics of a passive line-of-sight stabilization system with four-axis gimbal suspension," Russian Aeronautics (Iz VUZ), vol. 59, no. 4, pp. 480-488, 2017.

[19] A. N. Poo, C. L. Teo, M. K. Loh, and S. L. Tay, "Design and development of a two-axis passive line-of-sight stabilization system," Journal of Mechanical Design, vol. 117, no. 1, pp. 173-179, 1995.
[20] C. Wang, E. D. Burnham-Fay, and J. D. Ellis, "Real-time FPGA-based Kalman filter for constant and non-constant velocity periodic error correction," Precision Engineering, vol. 48, pp. 133-143, 2017.

[21] X. Gao, L. Zhao, J. Liu et al., "Real-time phase correction based on FPGA in the beam position and phase measurement system," IEEE Transactions on Nuclear Science, vol. 63, no. 6, pp. 2903-2908, 2016.

[22] M. H. Refan, A. Dameshghi, and M. Kamarzarrin, "Real-time differential global poisoning system stability and accuracy improvement by utilizing support vector machine," International Journal of Wireless Information Networks, vol. 23, no. 1, pp. 66-81, 2016.

[23] S. Lu, R. Cheng, K. Yang, Y. Zhu, L. Wang, and M. Zhang, "Real-time correction of periodic nonlinearity in homodyne detection for scanning beam interference lithography," Optical Engineering, vol. 57, no. 10, pp. 104107.1-104107.8, 2018.

[24] Y. U. Tao, H. U. Bing-Liang, G. A. Xiao-Hui, W. E. Ru-Yi, J. I. Juan-Juan, and H. O. Xiao-Hua, "Research on dynamic tracking and compensation method for hyperspectral interference imaging," Acta Photonica Sinica, vol. 45, no. 7, pp. 0710003.1-0710003.8, 2016.

[25] K. Xie, W. Liu, Q. Zhou, Z. Jiang, F. Xi, and X. Xu, "Real-time phase measurement and correction of dynamic multimode beam using a single spatial light modulator," Chinese Optics Letters, vol. 18, no. 1, pp. 11404.1-11404.5, 2020.

[26] M. Kim, C. Park, S. Je, H. Jang, C. Joo, and S. Kang, "Real-time compensation of simultaneous errors induced by optical phase difference and substrate motion in scanning beam laser interference lithography system," IEEE/ASME Transactions on Mechatronics, vol. 23, no. 4, pp. 1491-1500, 2018.

[27] H. Chen, B. Jiang, Z. Shi, Y. Sun, H. Song, and L. Tang, "Uncertainty modeling of the spatial coordinate error correction system of the CMM based on laser tracer multi-station measurement," Measurement Science and Technology, vol. 30, no. 2, 2019.

[28] O. KOSE and T. OKTAY, "Simultaneous quadrotor autopilot system and collective morphing system design," Aircraft Engineering and Aerospace Technology, vol. 92, no. 7, pp. 1093$1100,2020$.

[29] O. Kose and T. Oktay, "Investigation of the effect of differential morphing on forward flight by using PID algorithm in quadrotors," Journal of Aviation, vol. 4, no. 1, pp. 15-21, 2020.

[30] O. Kose and T. Oktay, "Lateral control with differential and collective morphing in quadrotors," Journal of Avation, vol. 4, no. 2, pp. 48-54, 2020.

[31] K. He, H. Hong, G. Jiang, and H. Gu, "Analysis of assembly error effect on stability accuracy of unmanned aerial vehicle photoelectric detection system," Applied Sciences, vol. 10, no. 7, p. 2311, 2020.

[32] H. L. Liu, M. Rasheed, and H. H. Younis, "A line measurement method for geometric error measurement of the vertical machining center," SN Applied Sciences, vol. 1, no. 4, p. 324, 2019.

[33] J. Zhong, S. Zhong, Q. Zhang, S. Liu, Z. Peng, and N. Maia, "Real-time three-dimensional vibration monitoring of rotating shafts using constant-density sinusoidal fringe pattern as triaxial sensor," Mechanical Systems and Signal Processing, vol. 27, no. 4, pp. 151-157, 2019.

[34] Y. Liu, G. Xiang, J. Guo, M. Zhou, and H. Liu, "Novel on-line north-seeking method based on a three-axis MEMS 
gyroscope," Journal of Bjing Institute of Technology, vol. 115, no. JAN.15, pp. 132-146, 2018.

[35] M. Rahman, J. Heikkala, and K. Lappalainen, "Modeling, measurement and error compensation of multi-axis machine tools. Part I: theory," International Journal of Machine Tools and Manufacture, vol. 40, no. 10, pp. 1535-1546, 2000.

[36] V. N. Yakimov and A. V. Mashkov, "Digital estimation of correlation function moments using analog-stochastic sign quantization of a random process," Measurement Techniques, vol. 59, no. 1, pp. 12-15, 2016.

[37] J. Boehm, A. Niell, P. Tregoning, and H. Schuh, "Global mapping function (GMF): a new empirical mapping function based on numerical weather model data," Geophysical Research Letters, vol. 25, 2006.

[38] S. H. Abaci, "Comparison of different order and heterogeneous residual variances Legendre polynomials in random regression models," Kafkas Universitesi Veteriner Fakultesi Dergisi, vol. 27, no. 1, pp. 1-6, 2021. 